HEAD AND NECK

\title{
Fibular osteofasciocutaneous flap in computer-assisted mandibular reconstruction: technical aspects in oral malignancies
}

\author{
Ricostruzione mandibolare con lembo osteocutaneo di fibula e programmazione \\ computer assistita: aspetti tecnici nei tumori maligni del cavo orale
}

\author{
M. BERRONE ${ }^{1}$, E. CROSETTI2, P.L. TOS ${ }^{3}$, M. PENTENERO ${ }^{4}$, G. SUCCO 5 \\ ${ }^{1}$ Department of Oncology, Phd Program in Experimental Medicine and Therapy, University of Turin, Italy; \\ ${ }^{2}$ FPO IRCSS, Candiolo Cancer Institute, Head \& Neck Oncology Unit, Candiolo, Turin, Italy; ${ }^{3}$ Microsurgery Unit, \\ Department of Orthopaedics and Traumatology, Orthopaedic and Trauma Center CTO, Turin, Italy; ${ }^{4}$ Department \\ of Oncology, Oral Medicine and Oral Oncology Unit, University of Turin, Italy; ${ }^{5}$ Department of Oncology, \\ Otolaryngology Unit, University of Turin, Italy
}

\section{SUMMARY}

Virtual surgical planning technology in head and neck surgery is witnessing strong growth. In the literature, the validity of the method from the point of view of accuracy and clinical utility has been widely documented, especially for bone modelling. To date, however, with its increased use in head and neck oncology, and consequently the increased need for bone and soft tissue reconstruction, is important to carry out the virtual programme considering not only bone reconstruction but also all aspects related to the reconstruction of soft tissue using composite flaps. We describe our approach to virtual planning in the case of composite flaps. The study reports six consecutive patients with malignant disease requiring mandibular bone and soft tissue reconstruction using fibular osteocutaneous flaps. In all six patients, the resection and reconstruction were planned virtually focusing on the position of cutaneous perforator vessels in order to schedule fibula cutting guides. There were no complications in all six cases. The technique described allowed us to schedule composite fibula flaps in mandibular reconstruction virtually with good accuracy of the position of the bone segment in relation to the cutaneous paddle, important for soft tissue reconstruction. Despite the limited number of cases, the preliminary results of the study suggest that this protocol is useful in virtual programmes using composite flaps in mandibular reconstruction. Further investigations are needed.

KEY WORDS: Fibular osteofasciocutaneous flap • Mandibular reconstruction • Virtual surgical planning • Computer-assisted mandibular reconstruction $\bullet$ Fibula harvestin

\section{RIASSUNTO}

L'utilizzo della pianificazione virtuale in chirurgia testa e collo è in forte crescita. In letteratura, la validità del metodo dal punto di vista dell'acuratezza e l'utilità clinica sono stati ampiamente documentati, in modo particolare per il rimodellamento osseo del lembo. Al giorno d'oggi, l'aumentato utilizzo della programmazione virtuale in chirurgia oncologica testa-collo e, conseguentemente, la maggiore necessità di ricostruzioni sia ossee che dei tessuti molli, rendono importante realizzare il programma virtuale considerando non solo la ricostruzione ossea, ma anche tutti gli aspetti relativi alla ricostruzione dei tessuti molli con lembi compositi. Descriviamo nel seguente articolo il nostro approccio alla pianificazione virtuale nel caso di lembi compositi. Lo studio riporta sei pazienti consecutivi con malattia maligna programmati mediante ricostruzione mandibolare computer assistita e lembi osteo-fascio-cutanei di perone. In tutti i sei pazienti, la resezione e la ricostruzione sono state progettate concentrandosi sulla posizione dei vasi perforanti cutanei, al fine di programmare la posizione più corretta delle guide di taglio a livello del perone in funzione della posizione dei vasi perforanti stessi. La tecnica descritta ci ha permesso di programmare lembi osteo-fascio-cutanei di perone nella ricostruzione mandibolare computer assistita, con buona precisione della posizione del segmento osseo rispetto alla padella cutanea, importante per la ricostruzione dei tessuti molli. Nonostante il numero limitato di casi, i risultati preliminari dello studio suggeriscono che questo protocollo è utile nella programmazione virtuale. Sono necessarie ulteriori indagini.

PAROLE CHIAVE: Lembo osteofaciocutaneo di perone $\bullet$ Ricostruzione mandibolare $\bullet$ Programmazione chirurgica virtuale $\bullet$ Ricostruzione mandibolare computer assistita

Acta Otorhinolaryngol Ital 2016;36:469-478 


\section{Introduction}

Tumours involving the mandible bone require complex reconstructive planning. In the late 1980s, the use of the fibula free flap (FFF) to reconstruct mandibular defects was described ${ }^{1}$ and over the following years, many authors have contributed to optimize the technique ${ }^{2-6}$. Today, this option is the gold standard for mandibular reconstruction in many centres. In particular, in extended bone and soft tissue resections, this flap can be modelled with multiple osteotomies and can provide bone, muscle and skin for composite reconstruction ${ }^{7}$. The surgical procedure is safe, even in elderly head and neck cancer patients ${ }^{8}$.

One of the most delicate aspects of mandibular reconstruction is the technique of bone modelling because of the non-linear nature of the mandibular bone. The risk of prolonging the period of ischaemia and not restoring correct bone-to-bone contact, and maxillo-mandibular and occlusal relationships, can ultimately lead to a higher rate of complications and poor aesthetic and functional results. In the past, stereolithographic models have helped immensely in surgical planning ${ }^{9-12}$ but recently, the introduction of computer-assisted mandibular reconstruction (CAMR) with its pivotal role in virtual surgical planning has further increased the accuracy of preoperative planning resulting in greater precision of the surgical procedure and reducing surgical time. CAMR requires careful planning in order to obtain the best oncological, functional and aesthetic outcome.

Virtual surgical planning technology in Head and Neck surgery is witnessing strong growth ${ }^{13}$. It is increasingly being adopted in many centres since a precise and functional reconstruction can be achieved with a vascularised fibula flap ${ }^{14-19}$ after tumour ablation as demonstrated in many studies ${ }^{20-22}$. Other benefits are improved facial appearance and function after dental rehabilitation, which must be considered an integral part of the reconstructive programme ${ }^{23-26}$ in order to improve the residual quality of life of cancer patients ${ }^{27}$. Moreover, this technology can significantly reduce operating time, especially in complex reconstructions.
One important aspect that we believe could be of interest to the scientific community is the scheduling of fibula cutting guides, their design, and intraoperative osteo-fascio-cutaneous flap management. In this paper, we discuss our experience with computer-assisted mandibular reconstruction (CAMR) using fibular osteo-fascio-cutaneous flaps in six patients. Our attention was focused on the correct positioning of the cutting guides, in order to reduce all possible mismatches with skin perforator vessels during composite flap harvesting.

\section{Materials and methods}

Six consecutive patients (three women and three men, mean age 61 years) were included in the study. They all suffered from malignant disease in the oral cavity requiring reconstruction with a fibular osteo-cutaneous flap previously planned with CAMR. The preoperative demographic data are summarised in Table I.

Informed consent was obtained from all patients. The work described has been carried out in accordance with the Declaration of Helsinki and approved by the authors' local institutional review board.

\section{Virtual surgical planning}

After the acquisition of a 64-slice high-resolution computed tomographic (CT) scan of the patient's craniofacial skeleton and angiographic CT scan of the lower legs (the donor site for bone and vessels), the Dicom data were sent to the modelling company. 3D rendering was performed using CMF software 6.1 (Materialise, Leuven, Belgium), which produces a three-dimensional model of both the mandible and the fibula. Before the web meeting with the biomedical engineers, the perforator vessels in the lower leg, in addition to the results of the previous assessment with angio CT, were identified using a hand-held Doppler and marked on the skin. The distance between the lateral malleolus and the perforator skin vessel was registered (Fig. 1a). Furthermore, preliminary measurements for the resective and reconstructive programme were made on the CT scan

Table I. Preoperative demographic data, planned fibular segments, skin island maximum diameter, distance between malleolus and skin perforating vessel, and ischaemia time.

\begin{tabular}{lllllllll} 
Sex & Age, years & Pathol & U.C. & Res, cm & \# seg & SP diam, cm & Distance M P, cm & Ischaemia time, min \\
M & 59 & SCC & BS & 8.859 & 2 & 7.0 & 14.5 & $60^{*}$ \\
F & 69 & SCC & BS & 7.759 & 2 & 8 & 10.7 & $65^{*}$ \\
F & 44 & SCC & B & 7.685 & 1 & 8 & 12.3 & 73 \\
F & 71 & SCC & BS & 7.064 & 2 & 6 & 11.2 & 73 \\
M & 57 & SCC & BSB & 11.669 & 3 & 10 & 13.6 & 80 \\
M & 69 & SCC & BS & 7.728 & 2 & 10 & 16.9 & 75 \\
\hline
\end{tabular}

Pathol: pathology affecting the mandibular bone; SCC: squamous cell carcinoma; U.C.: Urken classification of mandibular defects: C, condyle; R, ramus; B, body; SH, symphysis (half); S, symphysis; Res: length of planned resection (cm); \# seg: number of fibular segments planned for mandibular reconstruction; SP diam: skin paddle maximum diameter (cm); Distance M P: distance between malleolus and skin perforator vessel (cm); Ischaemia time: time calculated from pedicle detachment to flap revascularisation (min). 


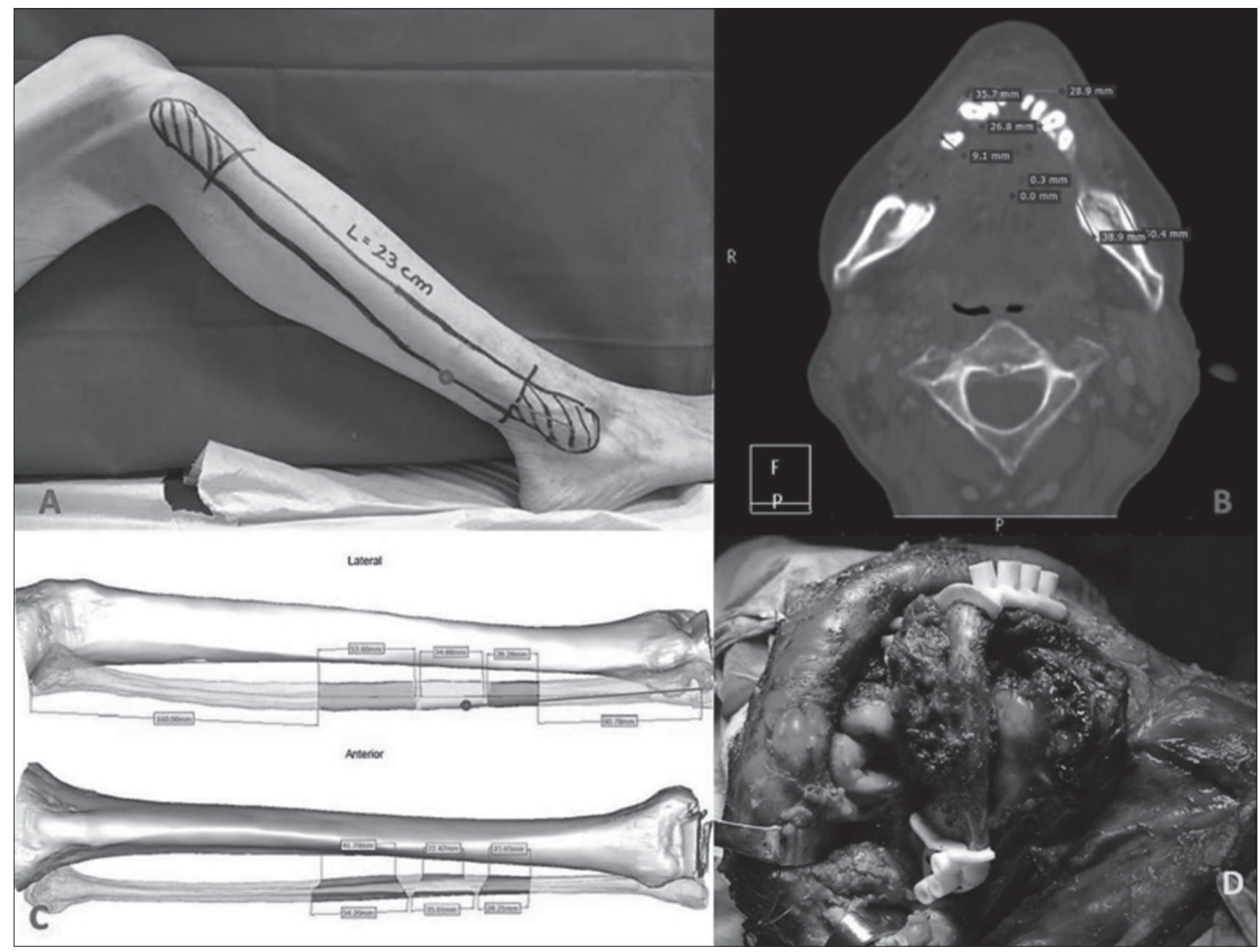

Fig. 1. a) Preoperative measurements of the distance between the malleolus and the perforator vessel; b) Preliminary measurements of the resective and reconstructive programme from CT scan; c) Reproduction of the distance between the malleolus and the perforator vessel on the virtual programme for fibula harvesting; d) Mandibular cutting guides.

(Fig. 1b). In all cases, the presence of the patient was scheduled during the course of the web meeting, in order to avoid any doubt if by chance any form of conflict had occurred between the planned resection and the position of the perforating vessels. Moreover, the presence of the patient is very important during the web meeting because this technology improves patient education and communication ${ }^{28}$.

During the web meeting between surgeons and biomedical engineers, the following were sequentially determined:

1. the amount of bone resection required (resulting from the clinical and radiological data, i.e. CT scan and MRI) allowing at least a $3 \mathrm{~cm}$ margin over the osteotomy to fix the patient-specific plate (PSP);

2. the side of the FFF (as a function of reconstructive requirement);

3. the type of reconstruction concerning the number of bone segments (segment length not less than $2.0 \mathrm{~cm}$ );

4. fibula and customised plate insetting and final position in relation to the remaining mandible and craniofacial skeleton;
5. the degree of obliquity of the osteotomies both on the mandible and fibula;

6. the number, position and inclination of screw holes both on the mandible (minimum of two on each mandibular stump) and fibula segments;

7. the shape and position of the cutting guides.

With an osteofasciocutaneous flap, the choice of the segment to be taken depends upon the measurements previously made in such a way as to have the skin perforator vessel in the most suitable position for soft tissue reconstructive purposes and which does not interfere with the osteotomy lines (Fig. 1c). A distance of $8 \mathrm{~cm}$ from the lower osteotomy to the ankle must be maintained for stability of the malleolar joint; proximally, a $7 \mathrm{~cm}$ bone segment is maintained to protect the peroneal nerve. Fibula cutting guides were all flange guides in order to be as compact as possible. The resection/reconstruction data were used to prepare autoclavable cutting guides to be used during mandibular resection and fibula harvesting as well as to model the final reconstructed mandible and the patient specific plate (PSP) (DePuy Synthes TRUMATCH ${ }^{\circledR}$ ). 

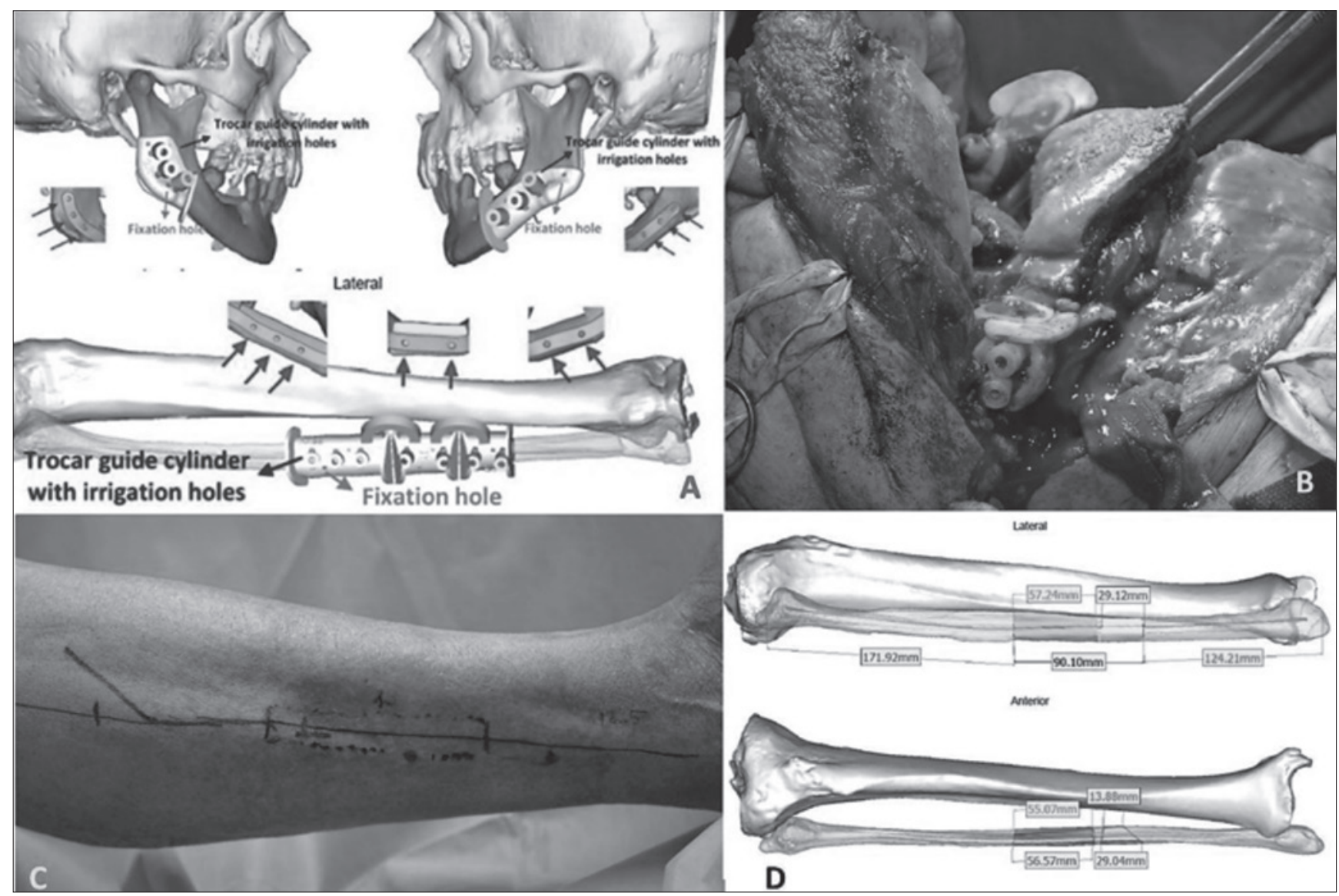

Fig. 2. a) Mandibular and fibular cutting guides were provided with fixation holes for temporary fixation and trocar guides for PSP fixation screws; b) Mandibular osteotomies completed with soft tissue resection; c) Preoperative measurements of virtual surgical planning and of the distance between the malleolus and the perforator vessel are drawn on the leg skin; d) Virtual surgical planning measurements.

Surgery

Access to the resection was via a visor flap. After neck dissection, access to the mandible was made and the cutting guides were secured in the planned position (Fig. 1d). Mandible and fibula cutting guides were provided with fixation holes for temporary fixation and trocar guides for PSP fixation screws (Fig. 2a); this planning allowed any type of pre-plating to be avoided. The osteotomies were completed with a sagittal or reciprocating saw following the cutting guides and the tumour was carefully removed. Normally, 45 degree osteotomies were planned and performed on both the mandible and fibula to obtain maximum bone-to-bone contact ${ }^{29}$. Finally, the operation was completed with soft tissue resection (Fig. 2b). Frozen sections were then taken on the mandibular periosteum, bone marrow, and soft tissues to check the radicality.

At the same time, a second surgical team proceeded to harvest the fibula. With the help of a hand-held Doppler and using the preoperative measurements of the distance between the malleolus and the perforator vessel, the position of the vessel itself was marked on the skin (Fig. 2c).
In addition, the virtual surgical planning measurements were annotated on the leg skin (Figs. 2c, d). An incision was made in the skin along the peroneus longus muscle, maintaining a distance of $2 \mathrm{~cm}$ from the posterior intermuscular septum, which could easily be palpated posteriorly to the muscle. According to the location of the perforator vessel found by preoperative mapping, the incision was curved slightly anteriorly in the region of the skin paddle. Despite the fact that mapping of the perforator vessels and thus positioning of the skin island is possible preoperatively using Doppler sonography, the skin paddle should not be designed until the cutaneous branches are clearly seen intraoperatively ${ }^{3031}$. In the standard situation, the skin paddle is centred vertically along the septum with its centre at the junction between the middle and lower third of the fibula. If only one perforator is enclosed, the flap size should not exceed $6 \times 10 \mathrm{~cm}^{31}$.

After identification of the perforator vessels and dissection, the fibular guides were secured to the bone to replicate the cuts for both the end and closing wedges in previously planned osteotomies (Fig. 3a). After po- 


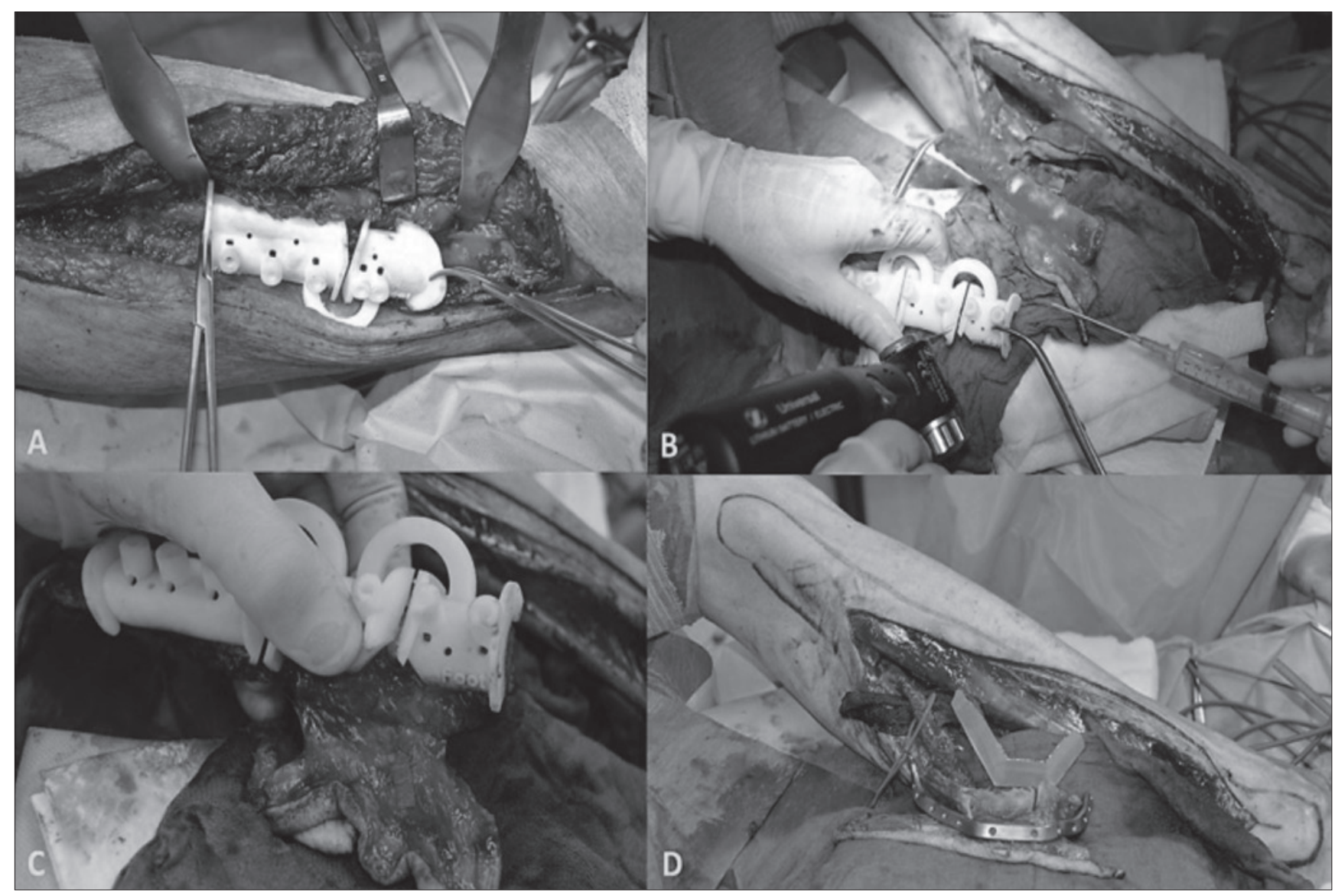

Fig. 3. a) Check of fibula cutting guides; b) Modification of the fibula cutting guides; c) Modified guide not interfering with perforator vessels; d) Fibula modelled before detaching the vascular pedicle.

sitioning and fixation of the cutting guides, the fibular distal and proximal osteotomies were performed with a margin of about $2 \mathrm{~cm}$, proceeding then to the final shaping upon receipt of the results of the frozen sections. This still retained the excess periosteum/fascia at the level of these margins to 'wrap' the jawbone creating a 'guide' for the ossification ${ }^{32}$. In two cases, there was interference between the cutting guide and the perforator vessel during the picking phase, and before fixing the template, we modified (minor correction of the flanges) the template itself so that it did not interfere with the vessel and did not create compression on the vessel itself (Figs. 3b, c). In two cases, the fibula was modelled and mounted on the patient specific plate (PSP) before detaching the vascular pedicle (Fig. 3d). The shaped fibula was then secured to the PSP in the planned position (Fig. 4a). The screw holes from the fibula cutting guides were designed to fit specific holes for the positioning and adaptation of the fibula with the PSP. The fibula and plate were then fixed to the native mandible with extremely precise bone-to-bone contact and positioning (Figs. $4 \mathrm{~b}, \mathrm{c}$ ). The fibular pedicle was then positioned and microvascular anastomosis was performed.

\section{Results}

In all patients, surgical margins (soft tissues and bones) were tumour-free. No flap failures occurred in any case, either for bone or cutaneous components. An unexpected sub-periosteal extension of the tumour occurred near the cutting line of the guide in only one case. In this case, considering that the periosteal extension occurred at the level of the alveolar process, a $2 \mathrm{~cm}$ stepwise enlargement of the mandible resection was carried out without changing the inferior amount of resection, where the positioning of the fibula was planned. In all other cases, no interference with the tumour was noted. Apart from two cases, there was a good correlation between the position of the perforating vessels and the bone segment to be taken. In the majority of cases, the skin paddle containing the vessels appeared positioned approximately at the level of the junction between the middle third and the lower third of the leg. High precision of cutting guides and a good fit of the PSP were found on both the mandible and fibula. 


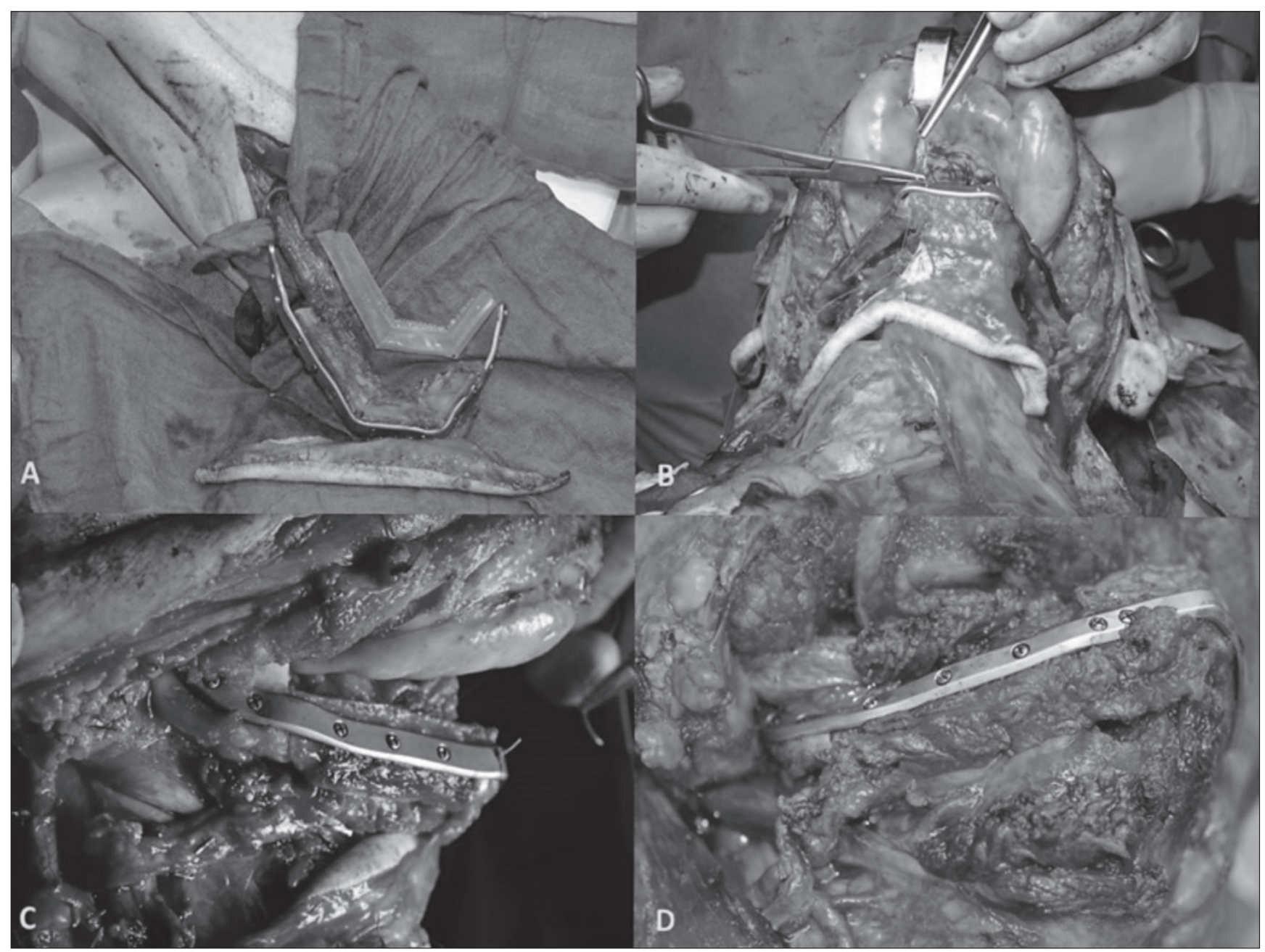

Fig. 4. a) Shaped fibula secured to the PSP in the planned position; b) Fibula and plate fixed to the native mandible; c) Extremely precise bone-to-bone contact and positioning; d) Extremely precise bone-to-bone contact.

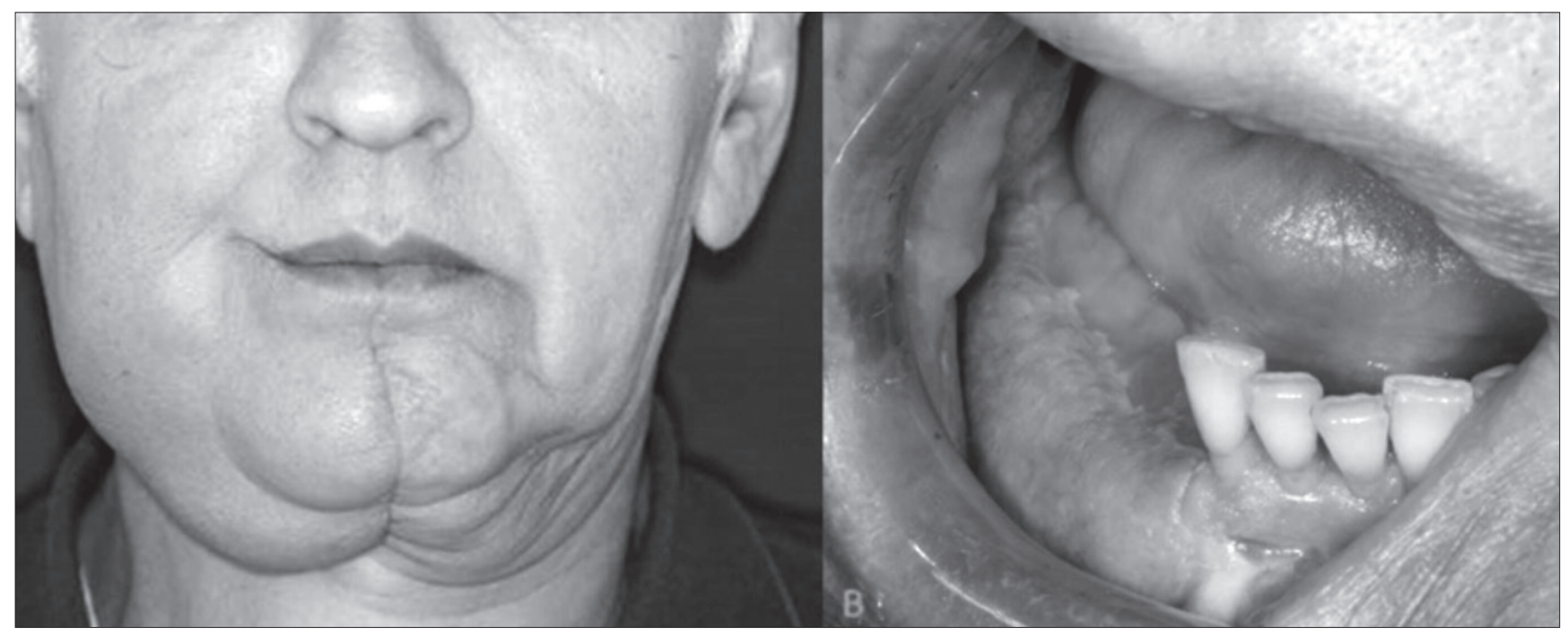

Fig. 5. a) Pleasant aesthetic final result; b) Good intraoral anatomy and morphology. 
Ischaemia time was recorded in all six cases, with an average of $71 \pm 7 \mathrm{~min}$. Excellent precision was also noted in bone-to-bone contact and position between the mandible and fibula grafts (Fig. 4c, d). In all six patients, a pleasant aesthetic final result was noted with good oral function (Figs. 5a, b).

\section{Discussion}

Virtual surgical planning technology in Head and Neck surgery is witnessing strong growth. In the literature, the validity of the method has been widely documented from the point of view of accuracy and clinical utility, especially for bone modelling. Moreover, as widely reported in the literature, this technology is especially useful in minimizing operating time, especially when multiple osteotomies are required. Computer-assisted mandibular reconstruction (CAMR) requires the resection to be scheduled with great precision, in advance of the time of surgery. To obtain maximum accuracy, it is first necessary to identify the extent of the tumour in the soft tissues, in particular the submucosal extent. Accurate knowledge of such extent, especially by annotating on radiologic pictures, allows surgeons to plan the most precise soft tissue resective programme during the virtual surgical planning process. Planning of bone resection is easier, because bone involvement by the tumour is easier to identify.

These decisions must be derived from a careful study of the clinical case, including palpation, as well as excellent quality, complete imaging. In practice, the 'real' time of the decision-making procedure is not at the operating table but during the simulation in the video conference, at which time, the whole team must have all the information needed to minimize a possible staging error. For this reason, we believe it is useful, at least in the early stages of the learning curve, to request the simultaneous presence of the patient during the web meeting. In at least a couple of cases, we have in fact taken steps to revisit the patient to dispel doubts about the true extent of the tumour and further control the position of the perforator vessels.

In order to increase the safety of the procedure, our policy foresees performing final osteotomies on the proximal and distal fibular segments when frozen sections of the mandibular periosteum, bone marrow and soft tissue have demonstrated the complete radicality of resection. To date, however, with the increased use of virtual surgical planning technology in Head and Neck oncology and, consequently, the increased need for bone and soft tissue reconstruction, it is important to carry out the virtual programme considering not only the bone reconstruction but also all aspects related to the reconstruction of soft tissue using composite flaps, and considering that functional outcome is more related to soft tissue reconstruction than to bone reconstruction. In fact, with this strategy, complex orofa- cial defects can also be reconstructed by adopting a single flap without involving more than one donor site ${ }^{33}$. On the other hand, more dissections are required and the placement of cutting guides on the fibula increases the possibility of a mismatch with perforating vessels during dissection. Therefore, an accurate preoperative knowledge of the perforators is required. In addition to the anatomical knowledge of the higher density of perforating vessels, represented by the skin overlying the lower one-third of the fibula ${ }^{3435}$, several imaging methods can help surgeons to detect perforator vessels including hand-held Doppler, colour Doppler ultrasound, computed tomography, and magnetic resonance angiography. In our experience, the use of the hand-held Doppler associated with CT angiography ${ }^{36}$ for the identification of the perforator vessels and manual measurement of the distance between the external malleolus and the vessel itself has proven to be a good method in CAMR with an osteocutaneous flap. It must be said, however, that the surgeon is ready to modify, even if minimally, the morphology of the guides if these interfere with the only perforating vessel ${ }^{37}$.

From these cases, we can conclude that there are essentially four critical points in the planning of computerassisted mandibular reconstruction with a composite free fibula flap in oral malignancies:

1) The potential discrepancy between the position of the perforator vessels for the skin paddle and the positioning of the cutting guides for bone shaping. It is clear that the Doppler study of the perforator vessels can undoubtedly be helpful even though often an imprecise concordance has been noticed. A second factor is the clear usefulness represented by the knowledge of the localised vascular anatomy of the lower limb, demonstrating how the majority of perforator vessels are located at the level of the lower to middle third of the fibula. Such positioning matches well with the need to obtain a vascular pedicle of the fibula of sufficient length to allow correct placement of the same after vascular anastomoses. Should there be a discrepancy between the positioning of the cutting guides and the position of the perforator vessel, the cutting guides can be moved, although not by much (a few centimetres), without compromising the reconstructive surgical planning. Should there be a mismatch between the cutting guide and the perforator flap, the guide could easily be modified to avoid such a phenomenon, without compromising it as the guiding template for the surgeon. 2) The difficulty in positioning the cutting guides on the fibula due to improper skeletonization of the bone on which the guide is placed. In this case, this would be a technical defect due to lack of knowledge of surgical timing of harvesting and the anatomy of the lower limb. The cutting guides per se, tend to be minimally invasive and are positioned on the external surface of the fibula where they do not impact either the pedicle or the perforator vessels. 3) The potential dis- 
crepancy between the effective extent of the tumour and the planned resection from which the preparation of the cutting guides for the mandible depends. Undoubtedly, the benefit derived from complete planning of the surgical procedure for both the extractive portion as well as the reconstructive one, could be nullified by an understaging that determines the need for a substantial increase in the resection area. On the other hand, the concern of finding oneself in this situation during surgery could paradoxically lead to planning of the bone resection as a systematic over-treatment. In our experience, direct assessment of the clinical and radiological data just prior to the web meeting, and in two cases, during the web meeting, would minimize such a risk. In the event that a planning defect is found, the resection can be increased providing that the comparative and orientation ratios with the cutting guides on the fibula are maintained (i.e. enlargement by $2 \mathrm{~cm}$ on the mandibular distal stud implies an enlargement of $2 \mathrm{~cm}$ on the fibular distal stud). For this reason, the fibula must be resected with at least 3-4 extra centimetres on both extremities and should not be modelled until the outcome of the frozen section is known. Particularly with the introduction of the PSP, which presents very precise design and characteristics, changes in the surgical programme are often not able to be revised, and for this reason, we always order the stereolithographic model of the mandible reconstruction from the modelling company in order to have the possibility of modelling a standard plate intraoperatively in the case of changes in the resective programme. 4) The extended planned timing of the surgery. This is undoubtedly a critical aspect that should be quantified and guaranteed. It is in advanced local tumours where a possible therapeutic delay of over a month could weigh heavily on the general treatment outcome, determining among other aspects, a discrepancy between the planned resection and the dimensions of the tumour at the time of surgery and also often an excessive weight loss because patients do not eat well and have pain. The timing objective between the moment of choice of treatment and the effective supply of the same should never exceed 4 weeks and this must be a fixed target.

\section{Conclusions}

The possibility of being able to always programme in the most accurate fashion the position of the bone segment in relation to the cutaneous island is nowadays a very important factor in order to obtain maximum accuracy in bone reconstruction, especially with the use of PSP. The application of CAMR in complex defects, where an osteofasciocutaneous flap is used for defect reconstruction, allows excessive handling of the flap itself to be avoided during the modelling phase. Moreover, thanks to virtual surgical planning, all the phases of fibula modelling and mounting on the reconstruction plate can be performed before detaching the vascular pedicle and this is a further advantage, especially in composite flap harvesting. The technique described in this article may be considered a valuable aid in the programming of composite flaps using virtual surgery, however, we agree with Deek and Wei ${ }^{38}$ that computer-aided surgery is an important but limited tool because of the many variables inherent in complex reconstruction that are not yet programmed into the computer algorithm.

When planning for soft-tissue reconstruction and coverage, osteoseptocutaneous fibula flap components, including the septocutaneous vessels and the intercomponent relationship, bone cross-section and nutrition, pedicle length, and surgical plan flexibility are important niches to be considered in virtual programmes ${ }^{38}$; however, this clashes with the accuracy of planning of the PSP plaques which, when intraoperative changes are required, these changes are difficult to instigate because the PSP present very precise design characteristics, and intraoperative changes in the surgical programme are often not able to be revised.

These considerations are reflected in the need for surgical teams to make a very precise diagnosis and accurate preoperative assessments so that the virtual surgical planning is highly predictable for both radical and reconstructive phases; moreover, the surgical team must be ready to change the reconstructive programme at every step with the help of traditional methods. Advances in radiological imaging for oral malignancies ${ }^{39} 40$ and perforator flaps ${ }^{41}$ will help surgeons to develop high-precision programmes for both radical and reconstructive phases. The future study and development of software able to fuse together $\mathrm{CT}$ and magnetic resonance angiography ${ }^{42}$ data are essential. Future research in the field of virtual surgical planning for soft tissue and tissue engineering will allow interventions to be planned that are increasingly predictable, both aesthetically and functionally. Further investigation is needed in this field.

\section{References}

1 Hidalgo DA. Fibula free flap: a new method of mandible reconstruction. Plast Reconstr Surg 1989;84:71-9.

2 Rana M, Warraich R, Kokemüller H, et al. Reconstruction of mandibular defects - clinical retrospective research over a 10-year period. Head Neck Oncol 2011;28:23.

3 Urken ML, Buchbinder D, Costantino PD, et al. Oromandibular reconstruction using microvascular composite flaps: report of 210 cases. Arch Otolaryngol Head Neck Surg 1998;124:46-55.

4 López-Arcas JM, Arias J, Del Castillo JL, et al. The fibula osteomyocutaneous flap for mandible reconstruction: a 15-year experience. J Oral Maxillofac Surg 2010;68:2377-84.

5 Wolff KD, Ervens J, Herzog K, et al. Experience with the 
osteocutaneous fibula flap: an analysis of 24 consecutive reconstructions of composite mandibular defects. J Craniomaxillofac Surg 1996;24:330-8.

6 Colletti G, Autelitano L, Rabbiosi D, et al. Technical refinements in mandibular reconstruction with free fibula flaps: outcome-oriented retrospective review of 99 cases. Acta Otorhinolaryngol Ital 2014;34:342-8.

7 Loeffelbein DJ, Holzle F, Wolff KD. Double-skin paddle perforator flap from the lateral lower leg for reconstruction of through-and-through cheek defect: a report of two cases. Int J Oral Maxillofac Surg 2006;35:1016-20.

8 Tarsitano A, Pizzigallo A, Sgarzani R, et al. Head and neck cancer in elderly patients: is microsurgical free-tissue transfer a safe procedure? Acta Otorhinolaryngol Ital 2012;32:371-5

9 Kernan BT, Wimsatt JA 3rd. Use of a stereolithography model for accurate, preoperative adaptation of a reconstruction plate. J Oral Maxillofac Surg 2000;58:349-51.

10 Lethaus B, Poort L, Böckmann R, et al. Additive manufacturing for microvascular reconstruction of the mandible in 20 patients. J Craniomaxillofac Surg 2012;40:43-6.

11 Salmi M, Paloheimo KS, Tuomi J, et al. Accuracy of medical models made by additive manufacturing (rapid manufacturing). J Craniomaxillofac Surg 2013;41:603-9.

12 Berrone M, Crosetti E, Succo G. Repositioning template for mandibular reconstruction with fibular free flaps: an alternative technique to pre-plating and virtual surgical planning. Acta Otorhinolaryngol Ital 2014;34:278-82.

13 Markey J, Knott PD, Fritz MA, et al. Recent advances in head and neck free tissue transfer. Curr Opin Otolaryngol Head Neck Surg 2015;23:297-301.

14 Foley BD, Thayer WP, Honeybrook A, et al. Mandibular reconstruction using computer-aided design and computeraided manufacturing: an analysis of surgical results. J Oral Maxillofac Surg 2013;71:e111-9.

15 Roser SM, Ramachandra S, Blair H, et al. The accuracy of virtual surgical planning in free fibula mandibular reconstruction: comparison of planned and final results. J Oral Maxillofac Surg 2010;68:2824-32.

16 Wang YY, Fan S, Zhang HQ, et al. Virtual surgical planning in precise maxillary reconstruction with vascularized fibular graft after tumor ablation. J Oral Maxillofac Surg 2016;74:1255-64.

17 Succo G, Berrone M, Battiston B, et al. Step-by-step surgical technique for mandibular reconstruction with fibular free flap: application of digital technology in virtual surgical planning. Eur Arch Otorhinolaryngol 2015;272:1491-501.

18 Tarsitano A, Ciocca L, Cipriani R, et al. Mandibular reconstruction using fibula free flap harvested using a customised cutting guide: how we do it. Acta Otorhinolaryngol Ital 2015;35:198-201.

19 Bolzoni A, Mapelli A, Baj A, et al. Evaluation of three-dimensional mandibular movements after reconstruction with free fibula flap. Acta Otorhinolaryngol Ital 2015;35:371-8.

20 Steinbacher DM. Three-dimensional analysis and surgical planning in craniomaxillofacial surgery. J Oral Maxillofac Surg 2015;73(Suppl 12):S40-56.

21 Kääriäinen M, Kuuskeri M, Gremoutis G, et al. Utilization of three-dimensional computer-aided preoperative virtual planning and manufacturing in maxillary and mandibular reconstruction with a microvascular fibula flap. J Reconstr Microsurg 2016;32:137-41.

22 Cornelius CP, Smolka W, Giessler GA, et al. Patient-specific reconstruction plates are the missing link in computer-assisted mandibular reconstruction: a showcase for technical description. J Craniomaxillofac Surg 2015;43:624-9.

23 Bak M, Jacobson AS, Buchbinder D, et al. Contemporary reconstruction of the mandible. Oral Oncol 2010;46:71-6.

24 Salinas TJ, Desa VP, Katsnelson A, et al. Clinical evaluation of implants in radiated fibula flaps. J Oral Maxillofac Surg 2010;68:524-9.

25 Qaisi M, Kolodney H, Swedenburg G, et al. Fibula jaw in a day: state of the art in maxillofacial reconstruction. J Oral Maxillofac Surg 2016;74:1284.e1-1284.e15.

${ }^{26}$ Freudlsperger C, Bodem JP, Engel E, et al. Mandibular reconstruction with a prefabricated free vascularized fibula and implant-supported prosthesis based on fully three-dimensional virtual planning. J Craniofac Surg 2014;25:980-2.

27 Petrosyan V, Ball D, Harrison R, et al. Among patients undergoing ablative treatment for oral cancer, does the provision of oral rehabilitation improve the quality of life? A review of the current literature. J Oral Maxillofac Surg 2016;74:1096. e1-1096.e12.

28 Pfaff MJ, Steinbacher DM. Plastic surgery applications using three-dimensional planning and computer-assisted design and manufacturing. Plast Reconstr Surg 2016;137:603e-16e.

29 Haddock NT, Monaco C, Weimer KA, et al. Increasing bony contact and overlap with computer-designed offset cuts in free fibula mandible reconstruction. J Craniofac Surg 2012;23:1592-5.

30 Wolff KD, Bauer F, Wylie J, et al. Peroneal perforator flap for intraoral reconstruction. Br J Oral Maxillofac Surg 2012;50:25-9.

31 Wolff KD, Hölzle F. Raising of microvascular flaps: a systematic approach. Berlin, Heidelberg, New York: Springer 2011.

32 Trignano E, Fallico N, Faenza M, et al. Free fibular flap with periosteal excess for mandibular reconstruction. Microsurgery 2013;33:527-33.

33 Kannan RY, Mathur BS, Tzafetta K. Single flap reconstruction for complex oro-facial defects using chimeric free fibular flap variants. J Plast Reconstr Aesthet Surg 2013;66:358-63.

34 Wei FC, Chen HC, Chuang CC, et al. Fibular osteoseptocutaneous flap: anatomic study and clinical application. Plast Reconstr Surg 1986;78:191-200.

35 Yu P, Chang EI, Hanasono MM. Design of a reliable skin paddle for the fibula osteocutaneous flap: perforator anatomy revisited. Plast Reconstr Surg 2011;128:440-6.

36 Ribuffo D, Atzeni M, Saba L, et al. Clinical study of peroneal artery perforators with computed tomographic angiography: implications for fibular flap harvest. Surg Radiol Anat 2010;32:329-34.

37 Yadav PS, Ahmad QG, Shankhdhar VK, et al. Skin paddle vascularity of free fibula flap - a study of 386 cases and a classification based on contribution from axial vessels of the leg. Indian J Plast Surg 2012;45:58-61. 
38 Deek NF, Wei FC. Computer-assisted surgery for segmental mandibular reconstruction with the osteoseptocutaneous fibula flap: can we instigate ideological and technological reforms? Plast Reconstr Surg 2016;137:963-70.

39 Blatt S, Ziebart T, Krüger M, et al. Diagnosing oral squamous cell carcinoma: how much imaging do we really need? A review of the current literature. J Craniomaxillofac Surg 2016;44:538-49.

40 Shintani S, Yoshihama Y, Ueyama Y, et al. The usefulness of intraoral ultrasonography in the evaluation of oral cancer. Int J Oral Maxillofac Surg 2001;30:139-43.

41 Kagen AC, Hossain R, Dayan E, et al. Modern perforator flap imaging with high-resolution blood pool MR angiography. Radiographics 2015;35:901-15.

42 Fukaya E, Saloner D, Leon P, et al. Magnetic resonance angiography to evaluate septocutaneous perforators in free fibula flap transfer. J Plast Reconstr Aesthet Surg 2010;63:1099-104.

Received: June 9, 2016 - Accepted: June 13, 2016

Address for correspondence: Mattia Berrone, Department of Oncology, University of Turin, Regione Gonzole 10, 10043 Orbassano (TO), Italy. E-mail: mattia.berrone@unito.it 